\title{
Classical research pathways and stem cell therapies
}

\begin{abstract}
The new field of regenerative medicine is in several ways controversial. The influence of political, economical and other factors non-related to evidence based in science complicate further the situation. This is a brief description of important aspects to consider towards improving the way we conduct research in medicine with special attention on the field of cell therapies.
\end{abstract}

Keywords: evidence based medicine, legislation, stem cell therapies, translational medicine
Volume I Issue 3 - 2016

Francisco Ruiz Navarro, Georg S Kobinia
Research Department,Austrian Society for Regenerative

Medicine, Austria

Correspondence: Francisco Ruiz-Navarro, Research Department, Austrian Society for Regenerative Medicine, Wolzeile 3/Top 2.I,Vienna,Austria, Tel +43676 42175 68, Email ruiznavarro.md@gmail.com

Received: June 03, 2016 | Published: June 16, 2016

\section{Introduction}

How stem cells therapies should be brought from the bench to the patient's bed occupied several discussions in the field of medicine. As the road of stem cell therapies has being paved with new discoveries, the legislative organs had been trying to implement research and treatment regulations that they consider adequate and that are suppose to be directed towards the safety of the patients. However, the scientific base of these regulatory implementations is not always clear and suddenly it seems that regulation of medical therapies has nothing to do with evidence-based medicine.

It is expected and understandable that treatments that do not fit into the classical pathways of a pharmacological compound provoke anxiety among legislators and legislated triggering disputes in all levels from the researchers to the patients. However, in this on-the road policy-making probably the best way to reduce misunderstanding or deviated intentions is correlating evidence, clinical needs, security and, most important, that it is directed towards improving the conditions of the patients.

In the ideal situation taking decisions about treatment approvals or how to conduct research should be in the first instance guided by scientific evidence and not by politics, economics or anxiety. Nevertheless, as in every other sphere of the society the financial interests play a central role. Therefore, it is prudent to ask us if the regulations more than being overprotective decisions of a worried regulatory organism are really a way of wealthy industries to maintain a comfortable position within the arising regenerative medicine market, mainly because these companies are the ones that can afford many years of investigations. On the other hand, it is also fair to ask if the pro-active leaders of the accelerating stem cell therapies bypassing current legislations are also guided by the financial benefits of implementing these therapies in their private practice or by a true intention to improve the patients' condition by offering the best possible treatment. In the worst case, existing advocates or oppositions are driven by their limited knowledge of the topic. Unfortunately, the intentionality of an act is impossible to know.

Important to stress is that, by no means, this should be understood as condemnation of a financial remuneration in exchange of providing a service either to direct treatments to patients or research and for the development of the treatments. The intention is more to stress the importance of regulating and acting according to what is most beneficial to the patient and until now we assume it is probably what evidence-based medicine dictate; otherwise we can go back 5,000 years and continue to treat all diseases with phlebotomies just because Hippocrates did it that way. Today, instead of hindering the medical knowledge development with magical thinking, we are hindering it with money and politics.

Keeping aside the obvious multi-interests that legislation brings, it is easy to think that legislation should be attached to scientific evidence. However, are we using the correct pathways to obtain scientific evidence in stem cell treatments? Furthermore, are the classical pathways still valid in the development of treatments in this new era of biotechnology, not only talking about stem cells? Or is the classical pathway - to obtain scientific evidence and getting a treatment approved hindering the medical developments?

\section{The current situation in medical research}

The best-case scenario is an experienced researcher that comes up with a new idea for a treatment. Usually the researcher is a basic scientist, not directly bounded to the clinical setting who has the function of a principal investigator in a wealthy University or company and is able to obtain financing relatively easy. This is probably an "ideal situation" if we do not take into account that in some point the research may have a small impact on the patients' everyday treatment. Assuming that this is a great idea and it is in the starting point under perfect circumstances, it needs around 20years, 350million dollars and to overcome a $95 \%$ probability of failing in preclinical stages in order to become available for patients in the clinical practice. ${ }^{1}$ It sounds difficult even for the ideal situation and it is also of a low percentage of the cases that have ideal environment. In this way, the main part of the ideas is in normal or under normal circumstances that decrease their probability of success.

Having this in mind, it is evident that as scientific community we are not really being efficient taking into account all the money and time spent compared to the low rate of evidence-based treatments that are used and that actually are modifying the clinical practice. Yes, there have been several important medical breakthroughs. No, it is not enough.

Some weak points are evident as a first bias of access to resources in which only those who can finance decades of research are the ones 
that control the field and of course the market, unfortunately these actors are not clinicians, and neither is they scientists. Added to this, as money has become the motor of academics, scientists are dependent on grants, sponsors, cooperation and the eagerness to obtain them has gone awry a lot of the objectives of research. It seems that priorities are shifting: from trying to get better treatments to searching for a good impact factor that guarantees academic position and more grants; from broader therapy options for patients to monopolize medicine.

The above mentioned is true for the classical pathways of treatments developing and of course the situation is permeating the field of regenerative medicine too.

\section{Cornerstone of evidence-based medicine}

The cornerstone of medical developments should be evidencebased medicine since we are trying to develop medicine away from the empiricism. This system graduates the level of evidence related to the consistency of the studies conducted. As good as it sounds; it seems to be just hypothetical. Up to date half of the treatments available in the regular practice do not have enough scientific evidence to support them, furthermore around $1 / 3^{\text {rd }}$ of them are proved to be ineffective or unsafe however all of them are approved by the regulatory agencies and used by medical doctors everyday. ${ }^{2}$ That means half of our medical practice is based on expert opinions, in other words empirical knowledge. It is not that the situation itself is wrong, it may be a hint that medicine is also about experience (even history has teach us the contrary). At the end discordancy arises; or developing treatments with evidence-based medicine is not working or the legislations are not attached to evidence or we are saying one thing and doing another.

Does the evidence-based medicine consider now that the ideal acquirement of knowledge is really effective to develop the best treatments to patients? Or we should adapt more to the clinical reality? Furthermore, we are still in baby steps to master this system of acquiring knowledge. We have to fight common challenges of other areas of science such as lack of negative results published, lack of reproducibility and the growing number of retractions. ${ }^{3}$

It is questionable whether we are getting closer to achieve significant evidence-based knowledge or if we are just becoming more inefficient in getting effective treatments to patients? It is easier to elucidate that this evidence-based pathway is effective in terms of knowing causes, diagnosis or pathophysiology (that should be the base of the treatments), however this is not reflected in the patients' care. Moreover, the new treatments, such as stem cells that are totally different to the classical conception of a pharmaceutical component are fating to follow the same development barriers.

If the effectiveness of developing treatments that cures or at least significantly change patient's life is not being achieved through the current way of developing medical knowledge. Maybe it is time to rethink and adjust shackles in the chain. Taking advantage of this necessity to implement special rules and systems for new treatments, we can also improve the classical way of research.

\section{Conclusion}

The medical practice seems to be more correlated to legislative decisions than based on scientific evidence and these legislations are based on several other factors, different and away from science. Furthermore, the way we are developing treatments is not efficient in terms of cost-benefit and objectives should be changed both for regular pharmaceutical compounds and especially for new biotechnology and biological therapies such as stem cell treatments in a way that patients' safety is not against broader option of treatments that can actually change the curse of the diseases.

The lack of adequate research may lead to a crisis in which no significant discoveries to treat patients will be achieved. This is mediated not only by external influences such as costs, market and politics but also because of the lack of communication between clinicians and basic researchers that limits the accurate direction of research towards patients.

The combination of maintaining classical ways of developing research in the medical field, together with the problems science is experiencing in several fields are harming all the medical areas but especially the ones that could make medical treatments look different in 50 years. The reasons vary but essentially it seems to be more of an interest that awakes finances, politics, personal interests and apprehension than patients.

The consequence besides a medical research system collapsing is that fields such as regenerative medicine are deteriorating (concerning the quality of research reflected in patients' treatments) and that is a field not even completely launched. The agitated environment, even expected, is dividing strengths and leaving the knowledge at the end together with the patient.

A different and adequate conception of how treatments should be developed is necessary and urgent. In the area of stem cell therapies lays the opportunity to develop a different approach to this specific field at the time we can come out with a new basis to do evidencebased medicine.

An empowerment of scientists, not just the ones in privilege spots, to decide routes of research together with the promotion of translational research, not just among basic and clinical science, but also among different areas of knowledge would open the panorama to develop new pathways.

\section{Acknowledgements}

None.

\section{Conflict of interest}

The author declares no conflict of interest.

\section{References}

1. Herper M. The Cost of Creating a new drug now $\$ 5$ Billion, pushing big pharma to change. Forbes/Pharma \& Heatlhcare. 2013.

2. Prasad V, Ioannidis JP. Evidence-based de-implementation for contradicted, unproven, and aspiring healthcare practices. Implement Sci. 2014;9:1.

3. Fanelli D. Negative results are disappearing from most disciplines and countries. Scientometrics. 2011;90(3):891-904. 